\title{
Meeting Koch's postulates for calcium signaling in cardiac hypertrophy
}

Commentary

See related article,

pages 1395-1406.

\author{
Kenneth R. Chien
}

University of California at San Diego (UCSD)-Salk Program in Molecular Medicine, UCSD School of Medicine, Basic Science Building 0613-C, 9500 Gilman Drive, La Jolla, California 92093, USA.

Phone: (858) 534-6835; Fax: (858) 534-8081; E-mail: kchien@ucsd.edu.

In 1890 , a country doctor in Germany revolutionized our thinking of human disease. The challenge of the day was to identify specific bacterial agents that were responsible for major disease outbreaks. Robert Koch, after careful study of disease epidemics from around the world, forged the precepts that unequivocally establish causality for a single infectious agent in the onset of a distinct disease (1). These "postulates" stated that: (a) the agent should be present in every case of the disease; (b) the agent must be isolated from the diseased host and grown in vitro; (c) the disease must be reproduced when the agent is delivered to a susceptible host; and (d) the agent should be recovered from the diseased animals. Koch's postulates formed the intellectual cornerstone for the next century of advances in microbiology and ultimately led to the discovery of the infectious basis for anthrax, cholera, and tuberculosis. In recognition of these seminal contributions, Robert A. Koch was awarded the 1905 Nobel Prize in Physiology and Medicine. As we enter a new century, it is becoming increasingly clear that the power of Koch's logic has extended beyond the boundaries of bacterial diseases. Although his views were based on the inoculation of agents involved in infectious diseases, the clarity of his thinking continues to guide a new generation of scientists who are using germline transmission in the mouse to establish causality for specific genes and pathways in the etiology of complex, acquired human heart diseases.

In this regard, the current article by Passier and colleagues is a valuable step toward fulfilling Koch's postulates, underscoring the potential importance of calcium signaling pathways in cardiac hypertrophy and failure (2). Alterations in calcium handling have long been known to be closely associated with the onset of cardiac hypertrophy and failure. However, only recently has there been substantial evidence to sup- port the notion that these changes in calcium might underlie pathways that contribute to the progression of either cardiac hypertrophy or failure. The discovery of calcium-calmodulin-dependent protein kinases (3) and the subsequent identification of their critical role in neuronal cell signaling (4) laid the groundwork for a critical examination of the role of CaM kinase-dependent signaling in in vitro cultured cardiac myocyte models of hypertrophy (5). By utilizing reporter genes that are activated during the hypertrophic response, it was subsequently shown that the $\delta$ isoform of CaM kinase II, which is translocated into the nucleus and is the predominant form of CaM kinase in the

which directly activates the protein via phosphorylation (11). The discovery of this CaM kinase pathway, which extends from the cytoplasm to the nucleus represents the major new finding of this paper (2). This report joins a large body of work that has previously focused on the role of a calcineurinNFAT signaling pathway in the control of the hypertrophic response, which appears to be a parallel calciumdependent pathway $(12,13)$. Coupling these data with other recent reports that have identified a critical role for calcium cycling defects in the progression of heart failure (14), evidence is mounting that calcium can provoke heart failure and hypertrophy (15). heart (6), was both necessary and sufficient to activate features of hypertrophy in vitro (7). By overexpressing a constitutively active form of CaM kinase IV specifically in the heart of transgenic mice under the control of the well-characterized $\alpha$-myosin heavy chain promoter (8), the study by Passier et al. now shows for the first time to our knowledge that the CaM kinase pathway is sufficient to activate many features of cardiac hypertrophy and failure in vivo (2). Using a lacZ indicator mouse line (9), the current study documents that one of the critical downstream targets in the CaM kinase pathway is the transcription factor, MEF-2, which had previously been shown to play a pivotal role in both skeletal and cardiac myogenesis (10). Previous studies in other cell types have documented that the nuclear transcription factor CREB is a major downstream target for CAM kinase,
This work is intriguing because of the efforts taken to extend previous in vitro observations to the in vivo context and the ability to make a new connection between cytosolic and nuclear signaling molecules. In particular, the use of the MEF-2 indicator line is ingenious (9), as it should ultimately allow the activation of this transcription factor to be monitored in the mouse during the biomechanical stress of pressure overload (16). As with all cutting-edge work, these studies of CaM kinase activation and cardiac hypertrophy suggest several other subsequent lines of experimentation. Given that CaM kinase IV is expressed at only trace levels in the heart (4), it will be of immediate interest to determine whether these observations can be extended to the predominant cardiac CaM kinase, the $\delta$ isoform of CAM kinase II, which is the predominant CaM kinase II activity in the heart (6). Recent- 
ly, two independent studies have shown that increasing the peak intracellular calcium transient can inhibit progression of heart failure in both genetic and acquired forms of cardiomyopathy (14, 17). In fact, ablating the endogenous brake on calcium cycling, phospholamban, can completely prevent the onset of cardiac hypertrophy and block the induction of atrial natriuretic factor in a mouse model of dilated cardiomyopathy (14). Because the decreased contractile function seen in the CaM kinase transgenic lines most likely reflects a decrease in the calcium transient, it will be important to characterize the compartmentalization of the calcium signal in the hypertrophied and failing heart. Doubly transgenic animals have proved difficult to generate from crosses between the CaM kinase and calcineurin mouse lines, most likely because the $\alpha$-myosin heavy chain promoter drives expression of both gene products at high levels in the fetal and the adult heart (18), leading to early lethality. For this reason, it will be critical to determine which aspects of the observed phenotypes in the CaM kinase transgenic line represent developmental effect on myocyte survival or morphogenesis and which derive from postnatal effects of the exogenous kinase. Finally, and perhaps most importantly, it will ultimately become necessary to determine whether the observed phenotypes reflect a role of the endogenous CaM kinase genes in the activation of biomechanical stress-induced hypertrophy, or if they arise in part from nonspecific effects of the 50- to 100-fold overexpression of a constitutively active protein.

With regard to this last point, there is a diverse and rapidly growing list of genes that can trigger features of hypertrophy and associated cardiomyopathy after their cardiac-specific expression (19-49) (Table 1), suggesting that multiple pathways can activate this complex adaptive response. As in the present study, in most of these cases, there are additional in vitro and in vivo data that support their role in the pathogenesis of hypertrophy. Accordingly, it is highly likely that these effects reflect a direct or indirect role for many of these genes in the hypertrophic response, a view that is supported by the fact that each of the cardiac phenotypes has distinct characteristics at the molecular, morphological, and physiological levels. However, discriminating between end points that arise strictly as a result of a signaling event from those that reflect nonspecific cardiac injury, presumably due to the disruption of the signaling stoichiometry in multiple pathways, could be a vexing problem. Death of cardiac myocytes may trigger not only postischemic heart failure, but also a transition between compensatory hypertrophy and dilated cardiomyopathy (50). As Izumo et al. (51) have shown, even the overexpression of the reporter gene GFP causes cardiotoxicity and cardiomyopathy when placed under the control of the $\alpha$ myosin heavy chain promoter. For this reason, the precise role of many of these putative hypertrophy genes may have been obscured by extraordinarily high levels of transgene expression.

A case in point is the large body of work, which has focused on the role of the calcineurin-NFAT pathway in cardiac hypertrophy $(12,13,33,52-54)$. Compelling data in transgenic mice (12), coupled with inhibition of the in vivo pressure overload response by cyclosporin, formed a cornerstone in support of a primary role for calcineurin as part of a final common pathway in hypertrophy (13). However, the story has grown increasingly complicated, as a number of groups have shown that cyclosporin fails to block the onset of pressure overload hypertrophy (55-58). The equivocal nature of the data raises the possibility that a portion of the in vivo effects observed in the calcineurin transgenics might be due to the nonspecific effects of a massive increase in cellular phosphatase levels. Supporting this notion, it was recently documented that NFAT3 knockout mice display a completely normal response to pressure overload hypertrophy, again raising the issue as to the precise role of the calcineurin pathway in the in vivo hypertrophic response (J.M. Leiden, personal communication). Recently, Tsao et al. reported a tenfold decrease in the level of calcineurin expression in the failing human heart (59). In addition, it has been noted that cyclosporin has little effect on in vivo cardiac hypertrophy in humans that receive chronic therapy during renal transplantation. However, cyclosporin is not an ideal agent to establish Koch's postulates for any specific pathway, given its pleiotropic effects. Of note, the calcineurin-NFAT pathway has also been shown to mediate skeletal muscle hypertrophy, indicating that components of this pathway may represent a conserved mechanism for hypertrophic signaling in other striated muscle cells. $(60,61)$. Taken together, it becomes apparent that although calcineurin may contribute to an important hypertrophic signaling pathway, its precise role in cardiac hypertrophy remains to be fully established. Definitive proof of causality awaits the engineering of a complete loss of calcineurin function specifically in heart cells. A similar caveat could apply to virtually all of the genes listed in Table 1.

The use of the mouse in the study of cardiac hypertrophy was initially envisioned as a tool to connect in vivo cardiac physiology with the role of specific genes, i.e., a direct means to examine whether single gene effects could activate all of the features of a hypertrophic response that would ordinarily be seen

\section{Table 1}

Transgenic models of cardiac hypertrophy ${ }^{A}$

Ligands

Receptors

Channels/Transporters

G proteins

Kinases

Phosphatases

Transcriptional factors

Sarcomeric or cardiac structural proteins

Sarcoplasmic reticular proteins

Other
TNF- $\alpha$; MCP-1; NGF $(32,39,41,43,49)$

$\beta$-2 Adrenergic receptors; angiotensin II type I receptor $(22,47)$

KV4.2; GLUT-1 (ref. 48; W. Dillman, personal communication)

Ras; RhoA; Gi; Gs; Gq; Gh (19, 20, 21, 23, 26, 33)

PKC 3 -2; P38; CaM Kinase IV (refs. 2, 44; Y. Wang, and K.R. Chien)

Calcineurin (12)

MYF5; NFAT; CREB; RXR $\alpha$; RAR $\alpha(12,24,38,42,46)$

$\alpha$-Myosin heavy chain; $\alpha$-tropomyosin; tropomodulin; Troponin T; myosin binding protein C; myosin light chain-1; myosin light chain-2 (25, 28-31, 34, 35, 37, 40, 45)

Calsequestrin (27)

Enteroviral genome, GFP $(36,51)$

APartial listing of mice which display cardiac hypertrophy after the cardiac-restricted overexpression of either wild-type or mutant forms of the proteins noted. 
in response to a complex stimulus, such as hypertension (for review, see ref. 62). The concept was modeled after the logic of Koch, with the thought that single gene effects might directly activate pathways that can specifically activate all of the features of the hypertrophic response. The past 5 years have seen an exponential growth in our understanding of this important adaptive response of the heart. However, along the way, it has become increasingly clear that hypertrophy is a multigenic, integrative response $(15,63)$. In addition, environmental and genetic modifiers may be more important in determining the phenotypic outcome than the effects of any single gene. In short, to unravel this complex physiological response of the heart, we may now need to move beyond Koch's postulates, because heart disease is often not initiated by a single stimulus or pathway. The challenge remains to identify the nodal points in the multiple pathways that govern critical functions of the cardiac muscle cell. Ultimately, it will become imperative to examine the consequences of the specific loss of function of these nodal genes in the setting of authentic, complex environmental stimuli that lead to hypertrophy: pressure overload, hypertension, postischemic injury, and hypoxia, among others. Refined single-cell physiological end points could be extremely useful, as we need to move beyond effects on single genes. Precision engineering of hypomorphic alleles, cell-type specific mutations, inducible mutagenesis, and genetic complementation should prove valuable in the next leg of the journey. There is an old Chinese saying that "a journey of a thousand miles begins with one step." For the next generation of cardiovascular physicians and scientists, half the fun will be getting there.

\section{Acknowledgments}

The author gratefully acknowledges valuable discussions with E. Olson and J. Leiden during the preparation of this Commentary. The author's laboratory is supported by grants from the National Institutes of Health, the Jean LeDucq Foundation, and an Endowed Chair from the California Affiliate of the American Heart Association.

1. Brock, T.D. 2000. Robert Koch: a life in medicine and bacteriology. ASM Press. Washington, DC, USA. 364 pp.

2. Passier, R., et al. 2000. CaM kinase signaling induces cardiac hypertrophy and activates the
MEF2 transcription factor in vivo. J. Clin. Invest. 105:1395-1406

3. Hanson, P.I., and Schulman, H. 1992. Neuronal $\mathrm{Ca}^{2+} /$ calmodulin-dependent protein kinases. Annu. Rev. Biochem. 61:559-601.

4. Braun, A.P., and Schulman, H. 1995. The multifunctional calcium/calmodulin-dependent protein kinase: from form to function. Annu. Rev. Physiol. 57:417-445.

5. McDonough, P.M., Stella, S.L., and Glembotski, C.C. 1994. Involvement of cytoplasmic calci$\mathrm{um}$ and protein kinases in the regulation of atrial natriuretic factor secretion by contraction rate and endothelin. J. Biol. Chem. 269:9466-9472.

6. Edman, C.F., and Schulman, H. 1994. Identification and characterization of $\delta_{\mathrm{B}}$-CaM kinase and $\delta_{\mathrm{C}^{-}}$CaM kinase from rat heart, two new multifunctional $\mathrm{Ca}^{2+} /$ calmodulin-dependent protein kinase isoforms. Biochim. Biophys. Acta. 1221:89-101.

7. Ramirez, M.T., Zhao, X., Schulman, H., and Brown, J.H. 1997. The nuclear $\delta_{\mathrm{B}}$ isoform of multifunctional $\mathrm{Ca}^{2+} /$ calmodulin-dependent protein kinase II regulates atrial natriuretic factor gene expression in ventricular myocytes. $J$. Biol. Chem. 272:31203-31298.

8. Gulick, J., Subramaniam, A., and Robbins, J. 1991. Isolation and characterization of the mouse cardiac myosin heavy chain genes. J. Biol. Chem. 266:9180-9185

9. Naya, F.J., Wu, C., Richardson, J.A., Overbeek, P., and Olson, E.N. 1999. Transcriptional activity of MEF2 during mouse embryogenesis monitored with MEF2-dependent transgene. Devel opment. 126:2045-2052.

10. Black, B., and Olson, E.N. 1998. Transcriptional control of muscle development by myocyte enhancer factor-2 (MEF2) proteins. Annu. Rev. Cell Dev. Biol. 14:167-196.

11. Sheng, M., Thompson, M.A., and Greenberg, M.E. 1991. CREB: a Ca ${ }^{2+}$ regulated transcription factor phosphorylated by calmodulindependent kinases. Science. 252:1427-1430.

12. Molkentin, J.D., et al. 1998. A calcineurindependent transcriptional pathway for cardiac hypertrophy. Cell. 117:215-228.

13. Sussman, M.A., et al. 1998. Prevention of cardiac hypertrophy in mice by calcineurin inhibition. Science. 281:1690-1693.

14. Minamisawa, S., et al. 1999. Chronic phospholamban-sarcoplasmic reticulum calcium ATPase interaction is the critical calcium cycling defect in dilated cardiomyopathy. Cell. 99:313-322

15. Chien, K.R. 1999. Stress pathways and heart failure. Cell. 98:555-558.

16. Rockman, H.A., et al. 1991. Segregation of atrial specific and inducible expression of an ANF transgene in an in vivo murine model of cardiac hypertrophy. Proc. Natl. Acad. Sci. USA. 88:8277-8281.

17. Miyamoto, M.I., et al. 2000. Adenoviral gene transfer of SERCA2a improves left-ventricular function in aortic-banded rats in transition to heart failure. Proc. Natl. Acad. Sci. USA. 97:793-798.

18. Morris, J.K., et al. 1999. Rescue of the cardiac defect in ErbB2 mutant mice reveals essential roles of ErbB2 in peripheral nervous system development. Neuron. 23:273-283.

19. Hunter, J., Tanaka, N., Rockman, H.A., Ross, J., Jr., and Chien, K.R. 1995. Ventricular expression of a MLC-2v-Ras fusion gene induces cardiac hypertrophy and selective diastolic dysfunction in transgenic mice. J. Biol. Chem. 270:23173-23178

20. Sah, V.P., et al. 1999. Cardiac-specific overexpression of RhoA results in sinus and atrioven tricular nodal dysfunction and contractile failure. J. Clin. Invest. 103:1627-1634.

21. Small, K., et al. 1999. Cardiac specific overex- pression of transglutaminase II $(\mathrm{G}(\mathrm{h}))$ results in a unique hypertrophy phenotype independent of phospholipase C activation. J. Biol. Chem. 274:21291-21296.

22. Rockman, H.A., et al. 1998. Expression of a beta-adrenergic receptor kinase 1 inhibitor prevents the development of myocardial failure in gene-targeted mice. Proc. Natl. Acad. Sci. USA. 95:7000-7005.

23. D’Angelo, D.D., et al. 1997. Transgenic Galphaq overexpression induces cardiac contractile failure in mice. Proc. Natl. Acad. Sci. USA. 94:8121-8126.

24. Subbarayan, V., et al. 2000. RXRalpha overexpression in cardiomyocytes causes dilated cardiomyopathy but fails to rescue myocardial hypoplasia in RXRalpha-null fetuses. J. Clin. Invest. 105:387-394.

25. Marian, A.J., et al. 1999. A transgenic rabbit model for human hypertrophic cardiomyopathy. J. Clin. Invest. 104:1683-1692.

26. Asai, K., et al. 1999. Beta-adrenergic receptor blockade arrests myocyte damage and preserves cardiac function in the transgenic G(salpha) mouse. J. Clin. Invest. 104:551-558.

27. Cho, M.C., et al. 1999. Defective beta-adrenergic receptor signaling precedes the development of dilated cardiomyopathy in transgenic mice with calsequestrin overexpression. J. Biol. Chem. 274:22251-22256.

28. Tardiff, J.C., et al. 1999. Cardiac troponin T mutations result in allele-specific phenotypes in a mouse model for hypertrophic cardiomyopathy. J. Clin. Invest. 104:469-481.

29. Muthuchamy, M., et al. 1999. Mouse model of a familial hypertrophic cardiomyopathy mutation in alpha-tropomyosin manifests cardiac dysfunction. Circ. Res. 85:47-56.

30. Welikson, R.E., et al. 1999. Cardiac myosin heavy chains lacking the light chain binding domain cause hypertrophic cardiomyopathy in mice. Am. J. Physiol. 276:H2148-H2158.

31. James, J., et al. 1999. Transgenic over-expression of a motor protein at high levels results in severe cardiac pathology. Transgenic Res. 8:9-22.

32. Franco, F., et al. 1999. Magnetic resonance imaging and invasive evaluation of development of heart failure in transgenic mice with myocardial expression of tumor necrosis factoralpha. Circulation. 99:448-454.

33. Mende, U., et al. 1998. Transient cardiac expression of constitutively active Galphaq leads to hypertrophy and dilated cardiomyopathy by calcineurin-dependent and independent pathways. Proc. Natl. Acad. Sci. USA. 95:13893-13898.

34. Oberst, L., et al. 1998. Dominant-negative effect of a mutant cardiac troponin $\mathrm{T}$ on cardiac structure and function in transgenic mice. $J$ Clin. Invest. 102:1498-1505.

35. Yang, Q., et al. 1998. A mouse model of myosin binding protein $\mathrm{C}$ human familial hypertrophic cardiomyopathy. J. Clin. Invest. 102:1292-1300.

36. Wessely, R., et al. 1998. Transgenic expression of replication-restricted enteroviral genomes in heart muscle induces defective excitation-contraction coupling and dilated cardiomyopathy. J. Clin. Invest. 102:1444-1453.

37. Tardiff, J.C., et al. 1998. A truncated cardiac troponin $\mathrm{T}$ molecule in transgenic mice suggests multiple cellular mechanisms for familial hypertrophic cardiomyopathy. J. Clin. Invest. 101:2800-2811.

38. Fentzke, R.C., Korcarz, C.E., Lang, R.M., Lin, H., and Leiden, J.M. 1998. Dilated cardiomyopathy in transgenic mice expressing a dominant-negative CREB transcription factor in the heart. $J$. Clin. Invest. 101:2415-2426.

39. Bryant, D., et al. 1998. Cardiac failure in transgenic mice with myocardial expression of tumor necrosis factor-alpha. Circulation. 97:1375-1381.

40. Sussman, M.A., et al. 1998. Myofibril degenera- 
tion caused by tropomodulin overexpression leads to dilated cardiomyopathy in juvenile mice. J. Clin. Invest. 101:51-61.

41. Kolattukudy, P.E., et al. 1998. Myocarditis induced by targeted expression of the MCP-1 gene in murine cardiac muscle. Am. J. Pathol. 152:101-111.

42. Colbert, M.C., et al. 1997. Cardiac compartment-specific overexpression of a modified retinoic acid receptor produces dilated cardiomyopathy and congestive heart failure in transgenic mice. J. Clin. Invest. 100:1958-1968.

43. Kubota, T., et al. 1997. Dilated cardiomyopathy in transgenic mice with cardiac-specific overexpression of tumor necrosis factor-alpha. Circ. Res. 81:627-635.

44. Wakasaki, H., et al. 1997. Targeted overexpression of protein kinase $\mathrm{C}$ beta2 isoform in myocardium causes cardiomyopathy. Proc. Natl. Acad. Sci. USA. 94:9320-9325.

45. Vikstrom, K.L., Factor, S.M., and Leinwand, L.A. 1996. Mice expressing mutant myosin heavy chains are a model for familial hypertrophic cardiomyopathy. Mol. Med. 2:556-567.

46. Edwards, J.G., et al. 1996. Cardiomyopathy in transgenic myf5 mice. Circ. Res. 78:379-387.

47. Paradis, P., Dali-Youcef, N., Paradis, F.W., Thibault, G., and Nemer, M. 2000. Overexpression of angiotensin II type I receptor in cardiomyocytes induces cardiac hypertrophy and remodeling. Proc. Natl. Acad. Sci. USA.
97:931-936.

48. Wickenden, A.D., et al. 1999. Targeted expression of a dominant-negative $\mathrm{K}(\mathrm{v}) 4.2 \mathrm{~K}(+)$ channel subunit in the mouse heart. Circ. Res. 85:1067-1076.

49. Heath, B.M., et al. 1998. Overexpression of nerve growth factor in the heart alters ion channel activity and beta-adrenergic signalling in an adult transgenic mouse. J. Physiol. (Lond.) 512:779-791.

50. Hirota, H., et al. 1999. Loss of a gp130 cardiac muscle cell survival pathway is a critical event in the onset of heart failure during biomechanical stress. Cell. 97:189-198.

51. Izumo, S. 2000. Signalling of cardiac hypertrophy. Proceedings of the Keystone Symposium on the Molecular Biology of the Cardiovascular System

52. Taigen, T., De Windt, L.J., Lim, H.W., and Molkentin, J.D. 2000. Targeted inhibition of calcineurin prevents agonist-induced cardiomyoctye hypertrophy. Proc. Natl. Acad. Sci. USA. 97:1196-1201.

53. Meguro, T., et al. 1999. Cyclosporin attenuates pressure-overload hypertrophy in mice while enhancing susceptibility to decompensation and heart failure. Circ. Res. 84:735-740.

54. Shimoyama, M., et al. 1999. Calcineurin plays a critical role in pressure overload-induced cardiac hypertrophy. Circulation. 100:2449-2454.

55. Zhang, W., et al. 1999. Failure of calcineurin inhibitors to prevent pressure-overload left ven- tricular hypertrophy in rats. Circ. Res. 84:722-728.

56. Ding, B., et al. 1999. Pressure overload induces severe hypertrophy in mice treated with cyclosporin, an inhibitor of calcineurin. Circ. Res. 84:729-734.

57. Luo, Z., Shyu, K.G., Gulaberto, A., and Walsh, K. 1998. Calcineurin inhibitors and cardiac hypertrophy. Nat. Med. 10:1092-1093.

58. Muller, J.G., Nemoto, S., Laser, M., Carabello, B.A., and Menick, D.R. 1998. Calcineurin inhibition and cardiac hypertrophy. Science. 282:1007.

59. Tsao, L., Neville, C., Musaro, A., McCullagh, K.J., and Rosenthal, N. 2000. Revisiting calcineurin and human heart failure. Nat. Med. 6:2-3.

60. Musaro, A., McCullagh, K.J., Naya, F.J., Olson, E.N., and Rosenthal, N. 1999. IGF-1 induces skeletal myocyte hypertrophy through calcineurin in association with GATA-2 and NFATcl. Nature. 400:581-585.

61. Semsarian, C., et al. 1999. Skeletal muscle hypertrophy is mediated by a $\mathrm{Ca}^{2+}$-dependent calcineurin signalling pathway. Nature. 400:576-581.

62. Chien, K.R. 1996. Genes and physiology: molecular physiology in genetically engineered animals. J. Clin. Invest. 97:901-909.

63. Hunter, J.J., and Chien, K.R. 1999. Signaling pathways for cardiac hypertrophy and failure. $N$. Engl. J. Med. 341:1276-1283. 